\title{
Well-being in the work environment as foundation to achieve sustainable development goal
}

\author{
Jekaterina Kuzmina ${ }^{1}$,Dzintra Atstāja ${ }^{1, *}$, Gundega Dambe ${ }^{2}$, Yaroslav Kichuk ${ }^{3}$, and \\ Valentyna Bykhovchenko ${ }^{4}$ \\ ${ }^{1}$ BA School of Business and Finance, $161 \mathrm{Kr}$.Valdemara, Riga, LV-1013, Latvia \\ ${ }^{2}$ Latvian Association for People Management, 45/47 Elizabetes, Riga, LV-1010, Latvia \\ ${ }^{3}$ Izmail State University of Humanities, 12 Repin str, 68600 Izmail, Ukraine \\ ${ }^{4}$ National University of Life and Environmental Sciences of Ukraine, 11 Heroiv Oborony str., 03041 \\ Kyiv, Ukraine
}

\begin{abstract}
Sustainable development of the world is one of the most discussed topics, while well-being and risk-awareness of the employees are important elements to achieve it. The aim of the study is to analyse the work environment factors that are considered important by prospective and new employees and compare them with the opinions of experienced professional HR and company managers. The second goal of the research paper is to measure the risk-awareness of young adults due to its relevance for the current discussion. The results of the study show that students consider safe and ergonomic working conditions, green thinking, flexible working hours and good pay as the most important factors in the workplace. HR professionals, on the other hand, consider the psychological climate, safety, engagement, and work-life balance as the most important factors. Young adults in Latvia show some level of risk-awareness, while a deeper understanding of the issues is necessary.
\end{abstract}

\section{Introduction}

Sustainable development is one of the most discussed topics in the last years, while due to the versatility of the issue the factors, goals, and methods of sustainability, as well as the ways to achieve and preserve it are still interesting research objects. In the year 2015, the United Nations General Assembly set a collection of 17 global Sustainable Development Goals (SDG), addressing the global social and economic issues including those related to poverty, inequality, health, education, climate, environmental degradation, prosperity, and peace and justice [1].

The 17 goals are built on the successes of the Millennium Development Goals, and they provide a guideline for the United Nations Development Programme to support governments to integrate the goals into national plans and policies. 8th SDG promotes inclusive and sustainable economic growth, as well as employment and decent work

${ }^{*}$ Corresponding author: dzintra.atstaja@ba.lv 
conditions. It emphasizes the reaching of higher productivity and growth rates, job creation, entrepreneurship, and innovation [2]. According to the recent study, the developed countries are showing a good level of progress, while the targets within the 8th SDG are almost met or very close to being met $[2,3]$. Nevertheless, it is essential to preserve the high success ratios, therefore, the discussion of well-being in the work environment and factors influencing it plays an essential role.

Employees' well-being is a widely discussed issue in the scientific literature. As suggested by International Labour Organization "Workplace Wellbeing relates to all aspects of working life, from the quality and safety of the physical environment, to how workers feel about their work, their working environment, the climate at work and work organization" [4].

Moreover, the document mentioned above underlines that it is essential to measure the well-being to ensure that employees are provided with safe and healthy working conditions, they are satisfied, and show a high engagement level. Furthermore, it is worth considering that well-being is one of the important factors defining an organization's long-term effectiveness. Last, but not least, the well-known evidence is the existence of the link between levels of employees ' productivity, overall health and well-being of the staff. To sum up, well-being and factors influencing it in the work environment are essential issues to be studied. Even though the topical issue is not new, but is less studied in Latvia, therefore the authors are willing to contribute to the current research, while discussing the point from two different perspectives: factors that are influencing young adults`well-being in the work environment and risk-awareness of young adults in Latvia in the context of well-being.

There are several reasons to concentrate on the young adults, and the authors are going to mention some: first, a large number - 14.7 thousand or $83 \%$ - of the population of Latvia in working age (15-62 years) went abroad looking for a better life and job conditions and opportunities. 9.5 thousand of them or $64 \%$ were $20-39$ years old. Second, ca. $62 \%$ of migrants of working age were 20-39 years old [5]. All the above factors highlight the necessity of retaining professionals. Third, young people are not only future employees but also potential entrepreneurs generating new ideas, building new companies, and creating new job opportunities.

It is worth considering that according to Global Entrepreneurship Monitor as for the year 2019 there still some areas to be improved, while both understandings of well-being factors and risk-awareness degree could help to do so. As calculated by the Global Entrepreneurship Monitor the perceived opportunities rate in Latvia is 35.6 vs. 53.7 Global average rate and 51.7 - Regional average rate - showing considerably lower \% of people who believe that there are opportunities to start a new business in the area where they live in comparison to the rest of the world, that would probably result in a lower rate of start-ups. On the other hand, there is a higher level of fear of failure (considering both legal and financial consequences of failure) that may prevent individuals from using business opportunities - 46.6 in Latvia vs. 41.7 - Global average rate and 40.1 - Regional average rate [6].

The purpose of the current research paper is to analyze the work environment factors that are considered as being significant by prospective and new employees (high school students) and compare them with the opinions of experienced professional HR and company managers. Assuming that there are some similarities in the perception of wellbeing factors from both parties. The authors decided to look at the factors through the riskmanagement framework and to study risk-awareness - the second goal of the study was to measure the risk-awareness of young adults (prospective and new employees, entrepreneurs) in Latvia. 


\section{Material and Methods}

The following section of the research paper is devoted to the methodology, it outlines how the research was designed, how the sample was selected, and how the data was collected and analyzed. Because the research consists of two following studies, each of them is described separately.

\subsection{Essay "My Ideal Workplace" and Interviews with HR Managers}

To find out the expectations of the new generation of employees towards the workplace, 15 students in the master study program and 20 students in the bachelor study program were asked to write an essay "My ideal workplace". The research was conducted in the period from October 2019 to February 2020. In total 35 essays were received, and descriptive and inferential statistical methods were used for their analysis. The obtained data were divided into groups - safety, well-being, and psychological climate. In December 2019, interviews were conducted with $30 \mathrm{HR}$ managers and specialists in safety. The purpose of the interviews was to determine the most significant factors influencing the well-being of employees, which are summarized in the word-cloud and analytically compared with students' opinions.

\subsection{Questionnaire about Risk-Awareness}

During the analysis of the results obtained by the study the authors were able to see the possibilities to continue the research by addressing the risk-awareness issue. Riskawareness could be defined as a base of the amount of knowledge and experiences, beliefs and values, as well as empathy and communication of the risks related to the organization's objectives and plans.

Moreover, the level of risk-awareness determines the capability to recognize risks, to evaluate and manage them. One of the significant questions within the scientific debate is risk culture and risk-awareness as they are both providing a positive input to the success and sustainability of the organization. The evidence was proven by several researchers [79].

The benefits of risk management were discussed by several authors [10-12] and were further considered in the current research. The second part of the research was conducted in the period from December 2020 to February 2021 - empirical research using the questioning technique to attain the answer from respondents to specific statements. Respondents' attitudes were based on the Likert's five-point scale, where 1 stands for "Strongly agree" and 5 means "Strongly disagree" with a statement. The questionnaire was completed by 121 respondents (aged from 19 to 29, students or employees with finished and unfinished higher education). To reach the goal of the study, the authors used the factor analysis to determine the relationships as described previously [13].

\section{Results and Discussion}

The following section of the research paper is devoted to the description and discussion of the results. It highlights the most significant findings from both studies and explains the connections between two relevant issues well-being and risk-awareness. 


\subsection{Well-being and Factors Influencing It}

The issues of well-being are a growing area of research, yet the question of how it should be defined remains unanswered [14]. Based on a Gallup study of more than 150 countries representing 98 percent of the world's population, Rath and Harter have identified five essential elements of life that transcend countries, faiths, and cultures. These elements include career well-being (How you occupy your time/liking what you do each day?), social well-being (Relationships and love in your life), financial well-being (Managing your economic life to reduce stress and increase security), physical well-being (Good health and enough energy to get things done daily), and community well-being (Engagement and involvement in the area where you live) [15]. Some of the mentioned elements the authors were able to determine by the conducted study. Analyzing the results obtained by the first study the authors were able to arrive at the following conclusions as described below.

While describing their ideal workplace young people - future employees are using several adjectives that could be divided into three big groups - safety, well-being, and psychological climate. It is worth considering that both safety at working place and psychological climate are factors determining well-being, but the students are using the word "well-being" with a slightly different connotation - broader meaning than it is the fact in the research literature.

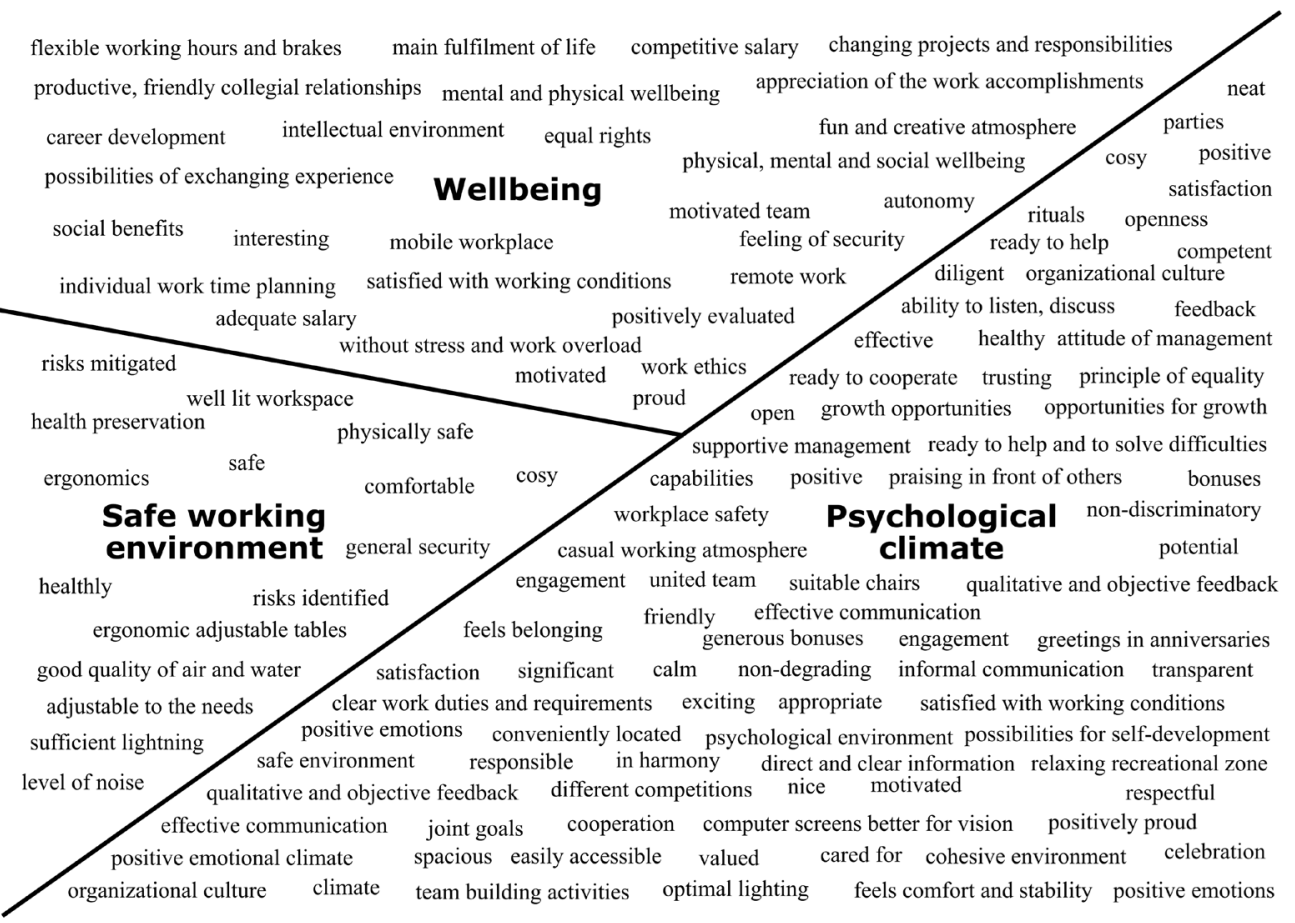

Fig. 1. The three dimensions of well-being.

Analysing the most commonly used adjectives for an ideal workplace regarding the safety criteria or factor the majority of students are using the following words as presented by Figure 1. Notably, safety is also attributed to both comfort and risk-management (the issue would probably be more important considering the current state of pandemics), while 
only one of the respondents suggests the level of noise. Analysing the most commonly used adjectives for an ideal workplace regarding the well-being criteria or factor the majority of students are using the following words as shown in Figure 1. It is possible to conclude that well-being is used as a combination of different factors, expectations, and personal values.

Around $90 \%$ of respondents are looking for flexible working hours - that could be considered as one of the major well-being components to evaluate the state of well-being at working place.

The results of the first study show that the students - future employees consider safe and ergonomic working conditions, green thinking, flexible working hours, and good pay as the most important factors influencing their well-being in the workplace. By interviewing the HR managers and safety specialists the authors arrived at slightly different results. The second group, on the other hand, consider the psychological climate, safety, engagement, and work-life balance as the most important factors determining the degree of the well-being of the employees.

The results gained in the study are broadly in line with conclusions drawn from the previous research outside Latvia. There is no doubt that high level of well-being in the work environment is good for the employees and the organization. It means lower sicknessabsence levels, better retention, and more satisfied customers. People with higher levels of well-being live longer, have happier lives and are easier to work with [16].

Personal well-being at work is influenced by many factors that are not directly workrelated. These factors are important, and it is crucial to recognize that what goes on at work is not the only thing that influences the well-being at work. Furthermore, it is equally important to recognize what actually goes on at work as it is generally the most important factor determining how people feel at work. Work-related factors are the most important contributors to well-being at work partly because they have a direct impact on well-being but also because it is easier for organizations to change and improve work-related factors. Improving someone's relationships with members of their family is not something that an organization might normally expect to be able to do - but improving relationships with someone's manager or colleagues is a different matter [17]. This chapter explains how the important ideas of employee engagement and personal well-being can be drawn together to provide a powerful combination to benefit employees and organizations alike. Around the world there is currently a great deal of interest in the concepts of employee engagement and employee well-being. The statistics of engagement are interesting and show that improving employee engagement leads to a range of positive outcomes for organizations - they also show that in many organizations the levels of engagement are actually quite low [18-21].

Also, according to the literature work-life balance is one of the important aspects, where workers are trusted enough to be able to work more flexibly and are able to invest time in their personal relationship outside work [22]. Similar conclusions were drawn from the current study.

\subsection{Risk-Awareness as Measured by Young Adults}

On the one hand, the results of the study allowed us to recognize that there are some differences in the expectations from the side of current or future employees regarding the ideal working place (ensuring employee well-being) and factors being considered as particularly important from HR management. On the other hand, one could identify particular similarities (even though expressed in different words) that should be highlighted and explained further. Both the parties are looking for the sustainable development of the employing organization. Young adults are expressing this aspect with the following wording as risks identified, risks mitigated, safety, management style, etc. HR managers are 
highlighting the importance of engagement of the employees and their involvement in the number of processes inside the organization.

Furthermore, it is important to deliberate that the accomplishment of sustainable development of the organization depends on the ability to minimize the potential losses in terms of capital, quality, human life, environmental and social damage, etc.

Consequently, risk management is a significant element of such a development - that was also recognized by young adults. The modern concept of risk management within the organization requires the engagement and co-operation of all the employees at different levels within their responsibilities' framework. Therefore, a high level of employee engagement leads to a range of positive outcomes for the organization. All these concepts mentioned above made the authors of the current research paper to study the risk-awareness of young adults to achieve the second goal of the study.

Figure 2 demonstrates how the factors influencing the well-being in the work environment are corresponding to the risk-awareness and risk management framework - in particular one could consider risk analysis and risk mitigation, financial and operational risks, portfolio approach to risk management.

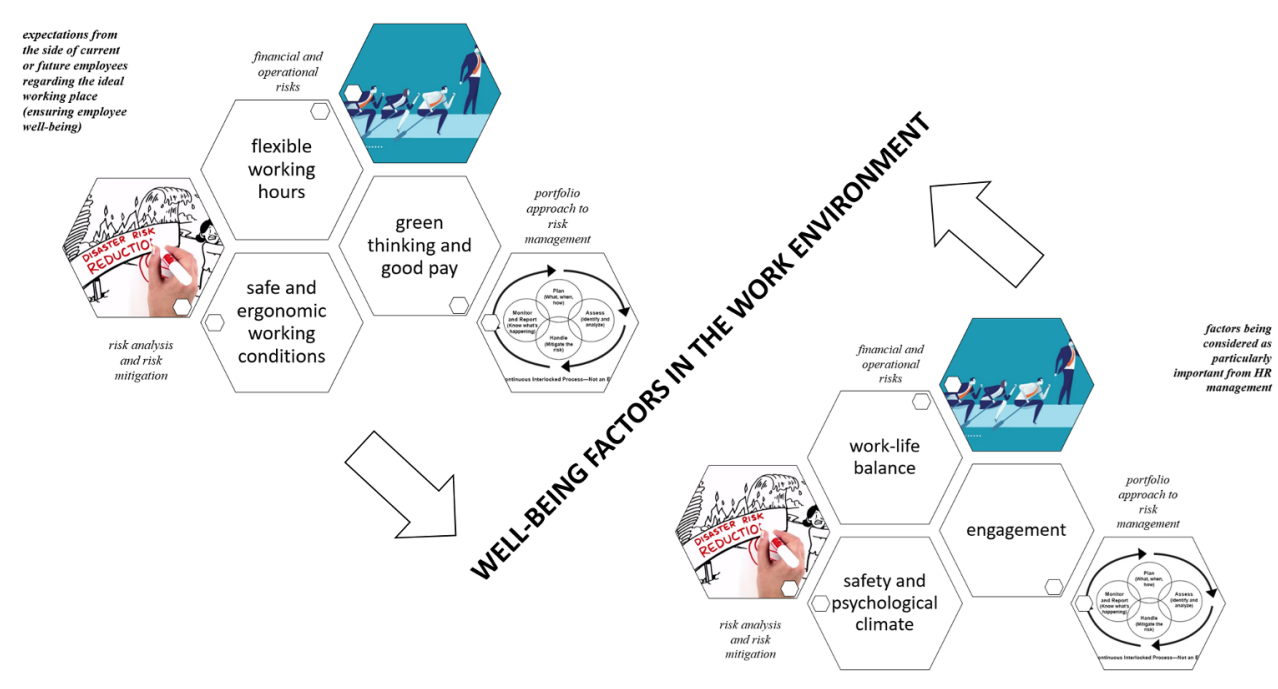

Fig. 2. Factors influencing the well-being within risk management framework.

The general description of the results of the survey is as following: $n=121$; average age is 21.8 years; female $(\%)-58.2$; full-time student $(\%)-49.6$; part-time students, employed $(\%)-32.2$ and entrepreneurs and employees (\%) - 18.2. The majority of the survey participants are recognizing the importance of risk management for the organization (above $4 / 5$ of the respondents) and the majority of them are overconfident about their knowledge level about risk management and the benefits of risk management (the average degree of personal knowledge is 2.89 on the scale from 5 - "very good" to - "very limited or no"), while the level of other members is assessed on average at 2.22. The existence of overconfidence could be probably explained by behavioural finance theory.

To determine the relationship between the attitude toward the risk management's influence on the perception of the future success of the organization the structural model was built assuming the independence of the variables and showing a sufficient level of statistical significance ( $p$-value equal to 0.0631 ). As a result, the following conclusions were gained: 
- The young adults demonstrate positive attitude toward the risk management as it contributes to the reduction of overall uncertainty of the business environment (model's coefficient -0.142 , significant at the 0.05 level).

- The young adults demonstrate positive attitude toward the risk management as it improves the reputation of the company among employees, clients, and other stakeholders (model's coefficient -0.076 , significant at the 0.05 level).

- The young adults demonstrate positive attitude toward the risk management as it protects company's resources reducing potential expenses and losses (model's coefficient 0.121 , significant at the 0.05 level).

- The young adults demonstrate positive attitude toward the risk management as it insures careful budgeting and lower cost of debt (model's coefficient -0.119 , significant at the 0.05 level).

The young adults demonstrate positive attitude toward the risk management as it helps to establish good communication culture and transparency of information flows within the organization (model's coefficient -0.009 , significant at the 0.05 level).

The results obtained show that risk management guarantees decrease of overall uncertainty and it has the most significant positive impact on a perception of the sustainable development of the organization. The particular benefit is in line with previous research, while the high level of significance of the factor for the young adults could be probably explained with psychological rather than economic issues.

The protection of the company's resources as well as prudent budgeting and lower cost of debt is the second significant risk management benefit that is acknowledged. This could be probably explained by the fact that these issues are usually highlighted during the trainings but should be explained in greater detail.

Astonishing results were achieved regarding the last factor - the establishment of good communication culture and transparency of information flows within the organization. Even though the respondents recognize its significance, but the level is rather low. The process of risk management to run smoothly requires the establishment of good communication culture within the company and the necessity for transparency of information flows to create the involvement and engagement of all the organization's employees. Based on the result achieved it is possible to claim that there are some shortages in the understanding of the risk management process.

The results achieved by the analysis of the data allow developing the following conclusions as well as describe next steps for future research. Young adults in Latvia are showing risk-awareness by recognizing the benefits of risk management for the future success of the business, nevertheless, the weight of each factor is different in the perception of the respondents. This could be attributed to the differences in the educational background, previous experience, or overall degree of knowledge [23]. Therefore, deeper understanding of the issues regarding risk management and sustainability is necessary, therefore additional efforts from the part of educational institutions and company`s management is required.

The framework of the current research did not use the further separation of respondents into groups that could probably highlight further questions and encourage the process to find the satisfactory explanation of differences if any are present. Moreover, the regional and cultural differences could be addressed and discussed.

\section{Conclusions}

Global sustainable development is a major issue, while the world's leaders agreed on a common sustainable route and numerous objectives and goals to attain this - the 17 SDGs, 
announced by the United Nations in 2015, to challenge economic, social and environmental issues and to encourage the concept of sustainability.

The current research is addressing in particular one of the 17 goals - the 8th and in this way is contributing both to the scientific discussion as well as providing specifics conclusions to consider for practical implementation to achieve global sustainable development.

The issues of well-being are a growing area of research, while practical implementations of the funding are of particular importance. The results of the study show that students - future workforce and creators of business - consider safe and ergonomic working conditions, green thinking, flexible working hours, and good pay as the most important factors in the workplace. The factors determined by the research are to some extent different from those mentioned by the HR professionals. Companies have to consider these differences. Moreover, the results of the study can help business leaders understand the importance of employee well-being and happiness at work in ensuring company sustainability and productivity in the future.

Risk management plays an important role in the day-to-day business of every organization, while risk-awareness is a co-stone of a successful risk management process. It is possible to claim that young adults in Latvia - potential entrepreneurs and owners of a business, as well as the current or future human capital - are showing risk-awareness, which could be seen as a positive sign. On the other hand, the respondents are overconfident about their knowledge, while a deeper understanding of the issues is necessary to achieve the sustainable development of the organization. The research findings presented in the current paper propose some guidance for organizations, HR managers, as well as higher education institutions willing to develop a better understanding of the risk management issues by the future and current employees to contribute to the long-term success of the organization and better world.

This research was carried out within the project of Latvian Council of Science Nr. lzp-2020/2-0317 «The Impact of COVID-19 on Sustainable Consumption Behaviours and Circular Economy.

\section{References}

1. United Nations (2015). The 17 goals. https://sdgs.un.org/goals

2. Halkos, G. \& Gkampoura, E.-Ch. (2021). Where do we stand on the 17 Sustainable Development Goals? An overview on progress, Economic Analysis and Policy, 70 https://doi.org/10.1016/j.eap.2021.02.001

3. United Nations (2020). Sustainable development goals progress chart 2020. https://sustainabledevelopment.un.org/content/documents/26727SDG_Chart_2020.pdf

4. International Labour Organization (n.d.). Workplace well-being, retrieved from https://www.ilo.org

5. Central Statistical Bureau of Latvia (2019). Official Statistics Portal. https://www.csb.gov.lv/en/statistics

6. Global Entrepreneurship Monitor (2019). Entrepreneurial Behaviour and Attitudes. https://www.gemconsortium.org/economy-profiles/latvia

7. Gatzert, N. \& Schmit, J. (2016) Supporting strategic success through enterprise-wide reputation risk management. The Journal of Risk Finance, $17(1), 26-45$. https://doi.org/10.1108/JRF-09-2015-0083

8. Lozano-Torró, A., García-Segura, T., Montalbán-Domingo, L., \& Pellicer, E. (2019) Risk Management as a Success Factor in the International Activity of Spanish Engineering. Administrative

Sciences, $\quad 9(1)$,

15. https://doi.org/10.3390/admsci9010015 
9. Amaechi, E. (2021) Understanding Culture and Success in Global Business: Developing Cultural and Innovative Entrepreneurs in Small Businesses. In Culture in Global Businesses 205-224. Palgrave Macmillan, Cham. https://doi.org/10.1007/978-3030-60296-3_9

10. Fatemi, A. \& Luft, C. (2002) Corporate risk management: costs and benefits. Global Finance Journal, 13(1). https://doi.org/10.1016/S1044-0283(02)00037-6

11. Sadgrove, K. (2016) The complete guide to business risk management. Routledge

12. Jain, P., Mannan, M. S., \& Pasman, H. J. (2020). Process system resilience: from risk management to business continuity and sustainability. International Journal of Business $\begin{array}{lllll}\text { Continuity and } & \text { Risk }\end{array}$ https://doi.org/10.1504/ijbcrm.2020.10027388

13. Brown, T.A. (2015). Methodology in the social sciences. Confirmatory factor analysis for applied research (2nd ed.). The Guilford Press

14. Dodge, R., Daly, A., Huyton, J., \& Sanders, L. (2012). The challenge of defining wellbeing. International Journal of Wellbeing, 2(3), 222-235. https://doi.org/10.5502/ijw.v2i3.4

15. Kruger, P. S. (2010). Wellbeing - The Five Essential Elements. Applied Research in Quality of Life, 6(3), 325-328. https://doi.org/10.1007/s11482-010-9127-1

16. Well-being: productivity and happiness at work. (2013). Journal of Public Mental Health, 12(3), 173-174. https://doi.org/10.1108/jpmh-10-2012-0010

17. Johnson, S., Robertson, I., \& Cooper, C. L. (2017). Work and Well-Being. In WELLBEING (pp. 89-108). Springer International Publishing. https://doi.org/10.1007/978-3319-62548-5 7

18. Johnson, S., Robertson, I., \& Cooper, C. L. (2017). Well-Being and Employee Engagement. In WELL-BEING (pp. 31-42). Springer International Publishing. https://doi.org/10.1007/978-3-319-62548-5 3

19. Yeshchenko, M., Koval, V., \& Tsvirko, O. (2019). Economic policy priorities of the income regulation. Espacios, 40 (38), 11.

20. Luchaninova, O., Koval, V., Deforzh, H., Nakonechna, L. \& Golovnia, O. (2019). Formation of communicative competence of future specialists by means of group work. Espacios, 40 (41), 11.

21. Petrova, M., Koval, V., Tepavicharova, M., Zerkal, A., Radchenko, A., \& Bondarchuk, N. (2020). The interaction between the human resources motivation and the commitment to the organization. Journal of Security and Sustainability Issues, 9(3), 897-907. https://doi.org/10.9770/jssi.2020.9.3(15)

22. Dewe, P., \& Cooper, C. (2012). Well-being and Work. Towards a Balanced Agenda, Palgrave Macmillan, US.

23. Arsawan, I. W. E., Sanjaya, I. B., Putra, I. K. M., \& Sukarta, I. W. (2018). The effect of expatriate knowledge transfer on subsidiaries' performance: a moderating role of absorptive capacity. In Journal of Physics: Conference Series, 953(1), 012082. 